\title{
Periodontal regenerative therapy
} CrossMark with enamel matrix derivative in the treatment
of intrabony defects: a prospective 2-year study

\author{
Fumi Seshima', Hideto Aoki ${ }^{1}$, Takahiro Takeuchi ${ }^{1}$, Eiichi Suzuki ${ }^{1}$, Daisuke Irokawa', Asako Makino-Oi', \\ Hiroki Sugito ${ }^{2,3}$, Sachiyo Tomita' and Atsushi Saito ${ }^{1,4^{*}}$ (1)
}

\begin{abstract}
Objective: To date, enamel matrix derivative (EMD) has been considered to be one of the few biomaterials for clinical use capable of demonstrating true periodontal regeneration. The aim of this two-center prospective clinical study was to evaluate 2-year outcome of periodontal regenerative therapy using EMD in the treatment of intrabony defects, performed as an 'advanced medical treatment' under the national healthcare system in Japan.

Results: Patients with chronic periodontitis who have completed initial periodontal therapy at either of the two dental school clinics were enrolled. Each contributed at least one intrabony defect of $\geq 3 \mathrm{~mm}$ in depth. During surgery, EMD was applied to the defect following debridement. Twenty-two participants (mean age 55.2 years old, 9 men and 13 women) completed 2-year reevaluation, and a total of 42 defects were subjected to data analysis. Mean gains in clinical attachment level (CAL) at 1 and 2 years were $2.9 \mathrm{~mm}$ (38\% of baseline CAL) and $3.1 \mathrm{~mm}(41 \%)$, respectively, both showing a significant improvement from baseline. There was also a significant reduction in probing depth (PD): mean reductions at 1 and 2 years were 3.2 and $3.3 \mathrm{~mm}$, respectively. There was a progressive improvement in the mean percentages of bone fill from $26 \%$ at 1 year to $36 \%$ at 2 years. No significant difference in CAL gain at 2 years was found between 3-wall bone defects and other defect types combined. In multiple regression analysis, the baseline PD was significantly associated with CAL gain at 2 years. In this population of patients, the treatment of intrabony defects with EMD yielded clinically favorable outcomes, as assessed by periodontal and radiographical parameters, over a period of 2 years.
\end{abstract}

Keywords: Periodontitis, Periodontal regeneration, Enamel matrix derivative

\section{Introduction}

Periodontitis is an inflammatory disease of tooth supporting tissues induced by dental plaque biofilm [1]. Cumulative evidence indicates that it is a dysbiotic disease which could induce a negative impact on systemic health [2]. In patients with moderate to advanced periodontitis, surgical periodontal therapy is often necessary, following non-surgical intervention.

Enamel matrix derivative (EMD) [3] has been used for the purpose of periodontal regeneration for over two

\footnotetext{
*Correspondence: atsaito@tdc.ac.jp

${ }^{1}$ Department of Periodontology, Tokyo Dental College, Tokyo, Japan Full list of author information is available at the end of the article
}

decades with favorable results [4-8]. It is considered to be one of the few biomaterials available for clinical use capable of histologically demonstrating true periodontal regeneration [8].

In Japan, under the system of 'advanced medical treatment', the use of treatment that is not covered by national healthcare insurance is permitted together with covered treatment in special cases. The use of EMD has been approved as an 'advanced medical treatment: bio-regeneration method' by the Ministry of Health, Labor and Welfare. This increased the opportunity for periodontitis patients to seek the regenerative therapy. Tokyo Dental College Chiba Hospital and Suidobashi Hospital received a formal approval for its use as the advanced medical 
treatment (on April 2008 and January 2011, respectively). Prior to this, we conducted a retrospective study evaluating the clinical outcome of treatment of intrabony defects with EMD [9]. We felt that it is our responsibility to longitudinally evaluate the regenerative therapy with EMD performed as the advanced medical treatment.

In this two-center prospective clinical study, we aimed to evaluate 2-year clinical outcome following surgical treatment of intrabony periodontal defects with EMD, performed as an 'advanced medical treatment' under the national healthcare system in Japan.

\section{Methods}

\section{Study design and participants}

This prospective, two-center, clinical study is part of our ongoing research on the longitudinal outcome of regenerative therapy using EMD alone, performed as an 'advanced medical treatment'. The participants were recruited from patients with chronic periodontitis [10], who visited Tokyo Dental College Suidobashi Hospital (Tokyo, Japan) or Tokyo Dental College Chiba Hospital (Chiba, Japan).

\section{Inclusion and exclusion criteria}

Inclusion criteria consisted of having interproximal sites with probing depth $(\mathrm{PD}) \geq 6 \mathrm{~mm}$, at least one intrabony defect $\geq 3 \mathrm{~mm}$ in depth in interproximal area of teeth and adequate level of plaque control (mean Plaque Index $\leq 1$ ) [11]. Participants must have received initial periodontal therapy.

Exclusion criteria were the presence of uncontrolled systemic diseases, smokers, allergy to common medications, concurrent or previous anti-resorptive agents such as bisphosphonate or other drug therapy, current pregnancy or lactation and contraindications for dental and/ or surgical interventions. Patients under 20 years old or with furcation involvements at target sites are excluded.

\section{Clinical examination}

The following parameters were recorded by trained, calibrated examiners at baseline essentially as described previously [12]: PD, gingival recession (GR), clinical attachment level (CAL), bleeding on probing (BOP), and tooth mobility (TM). Reevaluations were performed at 1 and 2 years after surgery.

\section{Radiographic assessment}

Semi-standardized radiographs were taken using film holders with customized occlusal stents as described previously [12]. Measurements from the radiographs were made using the Schei Ruler Technique [13]. The degree of change in the tooth axis heights between the cemento-enamel junction (CEJ) and bottom of the bone defect was defined as linear alveolar bone growth, and the percentage of bone fill was calculated by dividing the linear bone growth by the bone defect depth at baseline.

\section{Surgical procedures}

Following local infiltration anaesthesia, a full-thickness flap was used to gain access to the defect area. An effort was made to preserve papilla as much as possible during incision. Granulation tissue was removed, and scaling and root planning was performed. Following root conditioning and rinsing, 0.3 or $0.7 \mathrm{ml}$ EMD (Emdogain ${ }^{\circledR}$ Gel, BIORA AB/Straumann, Switzerland) was applied. No bone graft or other supplementary modalities were used. The flaps were then replaced and sutured with a PTFE non-resorbable sutures (Tefdesser II, 5-0, Kono Seisakusho, Chiba, Japan). No periodontal dressing was used. Eight periodontists with at least 3 years of periodontal training performed surgery.

The intrabony component of the defect (INTRA) was calculated as described previously [12]. The number of bone walls was also registered.

\section{Postsurgical care and maintenance}

The patients received antimicrobial agents (typically cefdinir $300 \mathrm{mg} /$ day, for 4 days). Standard analgesic was prescribed as necessary. They rinsed twice daily with an antimicrobial mouthrinse and started gentle wiping of the operated area with a soft toothbrush from the 3rd day.

The sutures were removed after 10-14 days. Professional supragingival tooth cleaning was performed at weeks 1, 2 and 4. Subsequently, all patients were placed on the maintenance program.

\section{Data management and statistical analysis}

Each patient contributed one to multiple defects. The primary endpoint variable was the change in CAL. Friedman test with Dunn's post hoc test was used to assess changes in data over time. Comparisons for BOP data were made by Fisher's exact test. Difference in CAL gain between two different defect types was sought by MannWhitney $U$ test. Correlation between variables was analyzed by Spearman's rank correlation. Multiple regression analysis was performed to determine contributions of the explanatory baseline variables. A software package (InStat version 3.10 for Windows, GraphPad Software, La Jolla, CA, USA) was used. Statistical significance was defined as a $p$ value of less than 0.05 .

\section{Results}

\section{Participants and baseline clinical parameters}

Twenty-two patients (mean age 55.2 years old, 9 men and 13 women) completed 2 -year reevaluation. Fortytwo defects were subjected to data analysis. Treated teeth comprised 9 incisors or canines (6 maxillary, 3 
mandibular), 9 premolars (4 maxillary, 5 mandibular) and 24 molars (11 maxillary, 13 mandibular).

No adverse events were observed in the participants throughout the study.

\section{Intrasurgical parameters}

The mean value for INTRA was $5.0 \pm 1.4 \mathrm{~mm}$. The defect type comprised the following: 1 and 3-wall combination: 2, 2-wall: 8, 2 and 3-wall combination: 8, 3-wall: 26.

\section{Change in clinical parameters}

At 1 year postoperatively, a significant improvement in CAL from baseline was observed $(p<0.01)$ (Table 1). An improvement from baseline was also observed at 2 years $(p<0.01)$. The mean gains in CAL (primary endpoint) at 1 and 2 years were $2.9 \pm 1.2 \mathrm{~mm}$ (range $0.0-6.0 \mathrm{~mm}$ ) and $3.1 \pm 1.3 \mathrm{~mm}$ (range $0.0-7.0 \mathrm{~mm}$ ), respectively. No significant difference in CAL gain was observed between at 1 and 2 years. Percentages of CAL gains at 1 and 2 years, relative to baseline CAL, were 38.1 and $40.7 \%$, respectively.

Distribution of CAL gain values at 2 years is shown in Fig. 1. CAL gain was noted at 41 sites and no change was found at one site.

Among the secondary endpoints, a significant improvement in PD from baseline was noted at 1 and 2 years (Table 1). No significant difference in PD was observed between at 1 and 2 years. Percentages of PD reductions at 1 and 2 years were 47.1 and $48.5 \%$, respectively. As for BOP, a significant difference from baseline was noted at 1 and 2 years. No significant difference from baseline in TM score was noted at 1 and 2 years.

At both 1 and 2 years, the contribution of GR to the PD reduction was minimal (Fig. 2).

\section{Assessment of bone fill}

At baseline, mean value of the percentage of bone loss was $47.5 \pm 13.3 \%$. The bone fill at 1 and 2 years

Table 1 Change in clinical parameters

\begin{tabular}{llll}
\hline Variable & Baseline & 1-year & 2-year \\
\hline $\mathrm{CAL}(\mathrm{mm})^{\mathrm{a}}$ & $7.6 \pm 1.8$ & $4.8 \pm 1.3^{* *}$ & $4.5 \pm 1.5^{* *}$ \\
& $(7.1-8.1)$ & $(4.2-5.3)$ & $(4.1-5.1)$ \\
$\mathrm{PD}(\mathrm{mm})^{\mathrm{a}}$ & $6.8 \pm 1.2$ & $3.3 \pm 1.0^{* *}$ & $3.2 \pm 1.0^{* *}$ \\
$\mathrm{BOP}($ mean \%) & $(6.4-7.1)$ & $(3.1-3.6)$ & $(3.0-3.4)$ \\
$\mathrm{TM}^{\mathrm{b}}$ & 42.9 & $2.3^{\#}$ & $9.5^{\#}$ \\
\hline
\end{tabular}

CAL clinical attachment level, $P D$ probing depth, BOP bleeding on probing, $T M$ tooth mobility

${ }^{* *} p<0.01$, significantly different from baseline, by Friedman test with Dunn's multiple comparisons test

\# $p<0.05$, significantly different from baseline, by Fisher's exact test

a mean \pm SD (lower 95\% confidence interval-upper 95\% confidence interval), ${ }^{b}$ mean $\pm S D$ postoperatively were $25.6 \pm 12.9$ and $36.2 \pm 14.6 \%$, respectively.

\section{Configuration of treated defects and CAL gain at 2 years}

When the values for CAL gain at 2 years were compared between 3-wall defects (contained defects) and others (1-wall, 2-wall, and combination defects), no significant difference was observed between groups $(p=0.13$, by Mann-Whitney U test) (Fig. 3).

\section{Relationship between CAL gain at 2 years and baseline variables}

A significant positive correlation was found with baseline PD and patient age (Additional file 1: Table S1). In multiple regression analysis, the baseline PD was significantly associated with CAL gain at 2 years (Table 2).

\section{Treatment case}

A representative case is shown in Additional file 2: Figure S1.
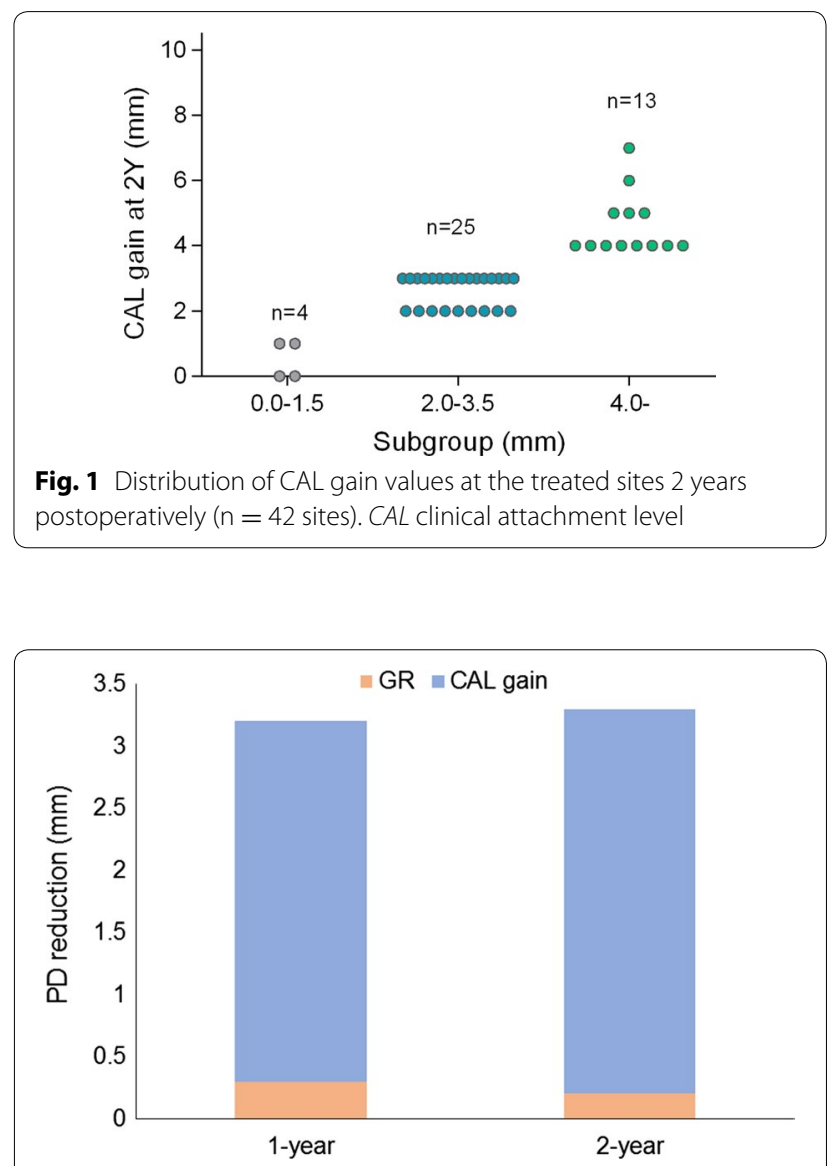

Fig. 2 Contribution of GR and CAL gain to PD reduction at 2 years. Mean values are shown. GR gingival recession, CAL clinical attachment level 


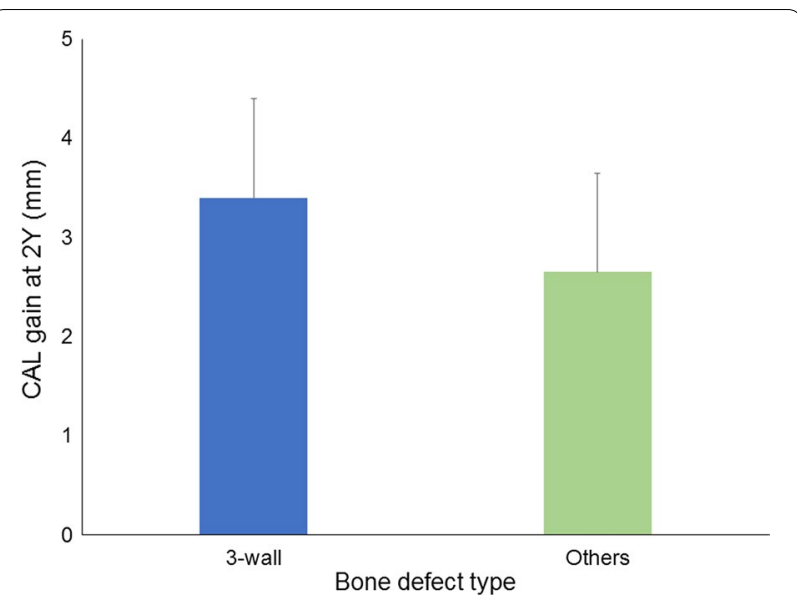

Fig. 3 Configuration of treated defects and CAL gain at 2 years. Data shown as mean $\pm \mathrm{SD}$. CAL clinical attachment level

Table 2 Multiple regression analysis for the association with CAL gain at 2 years

\begin{tabular}{lcrcc}
\hline Baseline variable & Coefficient & Confidence interval & $\boldsymbol{t}$ ratio & $\boldsymbol{p}$ \\
\hline CAL $(\mathrm{mm})$ & -0.032 & $-0.344-0.281$ & 0.206 & 0.838 \\
PD $(\mathrm{mm})$ & 0.508 & $0.004-1.011$ & 2.045 & 0.048 \\
TM & -0.385 & $-1.123-0.354$ & 1.058 & 0.297 \\
INTRA $(\mathrm{mm})$ & 0.260 & $-0.073-0.592$ & 1.584 & 0.122 \\
Patient age & 0.020 & $-0.014-0.053$ & 1.16 & 0.242
\end{tabular}

Dependent variable: $C A L$ gain at 2 years; $R^{2}=0.493$. Significant association is indicated in italics

CAL clinical attachment level, PD probing depth, TM tooth mobility, INTRA intrabony component

\section{Discussion}

In this study, we evaluated clinically the 2-year outcome of periodontal regenerative therapy using EMD alone, performed as an 'advanced medical treatment', in the treatment of intrabony defects. The EMD therapy yielded statistically significant gains in CAL and reductions in PD at 2 years, when compared with the preoperative data.

The mean value of the primary endpoint, CAL gain, was $2.9 \mathrm{~mm}$ at 1 year. This value is slightly smaller than the value of $3.2 \mathrm{~mm}$ reported in a meta-analysis on the management of 317 angular bone defects with EMD during an observation period ranging from 6 months to 1 year [14] and the value of $3.1 \mathrm{~mm}$ reported in a multi-center study [15]. At 2 years, the mean value was $3.1 \mathrm{~mm}$. This was comparable to that $(3.2 \mathrm{~mm})$ reported in our previous smaller-scale retrospective study [9] and other studies [15, 16].

A systematic review of the treatment of intrabony defects with EMD reported a significant additional gain in CAL of $1.3 \mathrm{~mm}$ compared with open-flap debridement, but no significant difference compared with resorbable membranes was shown [17]. In our previous study of the periodontal regenerative study using a deproteinized bovine bone mineral in combination with a collagen membrane [12], mean gain in CAL at 2.5 years was $1.4 \mathrm{~mm}$, which is smaller than the 2-year value in the present study. Although it is difficult to directly compare these data, it is remarkable that the EMD therapy yielded such result without the use of any bone substitutes.

In this study, the mean preoperative (baseline) PD value was $6.8 \mathrm{~mm}$. In the multiple regression analysis, baseline $\mathrm{PD}$ value was significantly associated with the CAL gain at 2 years. Similarly, other studies $[6,18]$ showed that deeper pockets gave significantly more CAL gain. These suggest that caution should be exercised when comparing the values of CAL gain in different studies.

Kitamura et al. [19] reported that the percentage of bone fill at 36 weeks following EMD therapy was 23\%, which was comparable to our 1-year value of $26 \%$. There was a progressive improvement in bone fill from at 1 year to $36 \%$ at 2 years. It has been reported that distinct radiographical bone fill was observed at as early as 5-6 months after the EMD therapy [20, 21]. Heijl et al. [20] also reported that further bone gain may be expected for as long as 3 years, which collaborates our findings.

Our multiple regression analysis showed that there was no significant association between INTRA and the CAL gain at 2 years. When the CAL gain values were compared between 3-wall defect (contained defect) and other defect types combined (uncontained defects), no significant difference was observed. Configuration of osseous defect has been shown to be an important determinant in EMD therapy [22]. Reflecting this, in a recent study of the use of EMD in the treatment of non-contained (1- and 2-wall) infrabony defects [23], the mean CAL gain at 1 year was shown to be $2.7 \mathrm{~mm}$. This CAL gain value is relatively modest, considering that the mean PD at baseline was $7.9 \mathrm{~mm}$, which was much greater than the value in this study. In our analysis, defect types other than 3-wall were combined due to the small sample size. It is necessary to evaluate the influence of bone defect configuration on longitudinal outcome of the use of EMD alone.

\section{Limitations}

In this study, sample size was relatively small. The study design was single-arm with no control group for direct comparison. The surgeries were performed by eight periodontists with various clinical experience levels.

\section{Additional files}

Additional file 1: Table S1. Correlations between baseline valuables and CAL gain at 2 years. r, Spearman coefficient. Significant differences are indicated in bold. CAL, clinical attachment level; PD, probing depth; TM, tooth mobility; INTRA, intrabony component. 
Additional file 2: Figure S1. A representative treatment case. 53-yearold woman with severe chronic periodontitis. a. Preoperative clinical view. PD $7.0 \mathrm{~mm}$, CAL $8.0 \mathrm{~mm}$, INTRA $6.0 \mathrm{~mm}$. b. During surgery; INTRA of the defect was $6.0 \mathrm{~mm}$. c. Preoperative (baseline) radiograph, in the distal aspect of the mandibular right second molar. Angular bony defect is evident. d. Radiograph after 1 year. An improvement in radiolucency can be observed in the distal aspect. e. Radiograph after 2 year, showing further improvement in the distal aspect. PD $4.0 \mathrm{~mm}$, CAL $4.0 \mathrm{~mm}$.

\section{Abbreviations}

EMD: enamel matrix derivative; CAL: clinical attachment level; PD: probing depth; BOP: bleeding on probing; TM: tooth mobility; GR: gingival recession; INTRA: intrabony component.

\section{Authors' contributions}

AS, AM-O, and ST contributed to study conception. FS and AS contributed to planning the study protocol, the analysis, interpretation of the data and drafting of the manuscript. HA, TT, ES, DI and ST contributed to the data collection. $\mathrm{FS}, \mathrm{HS}$, and AS contributed to data analysis. All authors read and approved the final manuscript.

\section{Author details}

${ }^{1}$ Department of Periodontology, Tokyo Dental College, Tokyo, Japan. ${ }^{2}$ Department of Operative Dentistry, Cariology and Pulp Biology, Tokyo Dental College, Tokyo, Japan. ${ }^{3}$ Department of Dental Hygiene, Tokyo Dental Junior College, Tokyo, Japan. ${ }^{4}$ Oral Health Science Center, Tokyo Dental College, Tokyo, Japan.

\section{Acknowledgements}

The authors thank the dental hygienists at Tokyo Dental College for their clinical efforts.

\section{Competing interests}

The authors declare that they have no competing interests.

\section{Availability of data and materials}

Data and further information about "Methods" section are available from the corresponding author.

\section{Consent to publish}

Written informed consent for publication was obtained from participants. All authors agreed to final submission.

\section{Ethics approval and consent to participate}

This study was performed according to the Helsinki Declaration, and the protocol was approved by the ethics committee of Tokyo Dental College (No. 550). Written informed consent for the participation in this study was obtained from each participant.

\section{Funding}

This study was self-funded by authors and their institution.

\section{Publisher's Note}

Springer Nature remains neutral with regard to jurisdictional claims in published maps and institutional affiliations.

Received: 25 May 2017 Accepted: 28 June 2017

Published online: 06 July 2017

\section{References}

1. Pihlstrom BL, Michalowicz BS, Johnson NW. Periodontal diseases. Lancet. 2005;366:1809-20.

2. Hajishengallis G. Periodontitis: from microbial immune subversion to systemic inflammation. Nat Rev Immunol. 2015;15:30-44.

3. Hammarström L. Enamel matrix, cementum development and regeneration. J Clin Periodontol. 1997;24:658-68.
4. Froum SJ, Weinberg MA, Rosenberg E, Tarnow D. A comparative study utilizing open flap debridement with and without enamel matrix derivative in the treatment of periodontal intrabony defects: a 12-month re-entry study. J Periodontol. 2001;72:25-34.

5. Trombelli L, Bottega S, Zucchelli G. Supracrestal soft tissue preservation with enamel matrix proteins in treatment of deep intrabony defects. J Clin Periodontol. 2002;29:433-9.

6. Tonetti MS, Lang NP, Cortellini P, Suvan JE, Adriaens P, Dubravec D, et al. Enamel matrix proteins in the regenerative therapy of deep intrabony defects: a multicenter randomized controlled clinical trial. J Clin Periodontol. 2002;29:317-25.

7. Yilmaz S, Kuru B, Altuna-Kirac E. Enamel matrix proteins in the treatment of periodontal sites with horizontal type of bone loss. J Clin Periodontol. 2003;30:197-206

8. Miron RJ, Sculean A, Cochran DL, Froum S, Zucchelli G, Nemcovsky C, et al. Twenty years of enamel matrix derivative: the past, the present and the future. J Clin Periodontol. 2016;43:668-83.

9. Fujinami K, Hayakawa H, Ota K, Ida A, Nikaido M, Makiishi T, et al. Two-year follow-up of treatment of intrabony periodontal defect with enamel matrix derivative. Bull Tokyo Dent Coll. 2011:52:215-21.

10. Armitage GC. Development of a classification system for periodontal diseases and conditions. Ann Periodontol. 1999;4:1-6.

11. Silness J, Löe H. Periodontal disease in pregnancy II. Correlation between oral hygiene and periodontal condition. Acta Odontol Scand. 1964;22:121-35.

12. Irokawa D, Takeuchi T, Noda K, Goto H, Egawa M, Tomita S, et al. Clinical outcome of periodontal regenerative therapy using collagen membrane and deproteinized bovine bone mineral: a 2.5-year follow-up study. BMC Res Notes. 2017;10:102.

13. Schei O, Waerhaug J, Lovdal A, Arno A. Alveolar bone loss as related to oral hygiene and age. J Periodontol. 1959;30:7-16.

14. Kalpidis CDR, Ruben MP. Treatment of intrabony periodontal defects with enamel matrix derivative: a literature review. J Periodontol. 2002;73:1360-76.

15. Sculean A, Schwarz F, Miliauskaite A, Kiss A, Arweiler N, Becker J, et al. Treatment of intrabony defects with an enamel matrix protein derivative or bioabsorbable membrane: an 8-year follow-up split-mouth study. J Periodontol. 2006;77:1879-86.

16. Heden $G$, Wennström JL. Five-year follow-up of regenerative periodontal therapy with enamel matrix derivative at sites with angular bone defects. J Periodontol. 2006;77:295-301.

17. Koop R, Merheb J, Quirynen M. Periodontal regeneration with enamel matrix derivative in reconstructive periodontal therapy: a systematic review. J Periodontol. 2012;83:707-20.

18. Zucchelli G, Bernardi F, Montebugnoli L, De Sanctis M. Enamel matrix proteins and guided tissue regeneration with titanium-reinforced expanded polytetrafluoroethylene membranes in the treatment of infrabony defects: a comparative controlled clinical trial. J Periodontol. 2002;73:3-12.

19. Kitamura M, Akamatsu M, Kawanami M, Furuichi Y, Fujii T, Mori M, et al. Randomized placebo-controlled and controlled non-inferiority phase III trials comparing trafermin, a recombinant human fibroblast growth factor 2, and enamel matrix derivative in periodontal regeneration in intrabony defects. J Bone Miner Res. 2016;31:806-14.

20. Heijl L, Heden G, Svardstrom G, Ostgren A. Enamel matrix derivative $\left(\right.$ Emdogain $^{\circledR}$ ) in the treatment of intrabony periodontal defects. J Clin Periodontol. 1997;24:705-14.

21. Saito A, Hayakawa H, Ota K, Fujinami K, Nikaido M, Makiishi T. Treatment of periodontal defects with enamel matrix derivative: clinical evaluation at early healing stages. Bull Tokyo Dent Coll. 2010;51:85-93.

22. Froum S, Lemler J, Horowitz R, Davidson B. The use of enamel matrix derivative in the treatment of periodontal osseous defects: a clinical decision tree based on biologic principles of regeneration. Int J Periodontics Restorative Dent. 2001;21:437-49.

23. Losada M, González R, Garcia ÀP, Santos A, Nart J. Treatment of noncontained infrabony defects with enamel matrix derivative alone or in combination with biphasic calcium phosphate bone graft: a 12-month randomized controlled clinical trial. J Periodontol. 2017;88:426-35. 